\title{
Design and Implementation of the System of Intelligent Traffic Light
}

\author{
Limin $\mathrm{Xu}$ \\ North China Electric Power University, Beijing, 102206, China
}

\begin{abstract}
At present, China's economy is developing rapidly, and the problem of traffic congestion is becoming more and more serious. This paper designs an intelligent traffic light system, which can effectively alleviate the traffic inconvenience. Firstly, the paper introduces the whole design idea of the traffic light system, and then designs the three modules of the traffic system, including the vehicle detection module, the traffic control module and the signal display module.The simulation results show that the system can provide the intelligent technical solutions for the urban traffic light control system.
\end{abstract}

Keywords:intelligent traffic light, control system, design and implementation

\section{Introduction}

With the rapid development of social economy and urban traffic, traffic has become the solution to ensure the orderly, safe and fast operation of urban life. On the other hand, as a kind of automatic control device based on microcomputer technology,programmable logic control has been widely used in various fields, such as machinery manufacturing, metallurgy, chemical industry, energy and so on. Integration and intelligence is the direction of development and research of city traffic signal control system, however, congestion management control structure for complex characteristics of traffic system scale and the city traffic bottlenecks and represents intelligence plan is the intelligent traffic control optimization key management. The lifeblood of our city's economic activities is the traffic. It has a very important role in the economic development of our city and the improvement of people's living standard. Urban traffic is an important factor that restricts the development of the city and the economic construction of 
the city. The infinite increase of city road length and limited increase of vehicles plus the two contradictions is the ultimate cause of traffic congestion in the city. The increasing traffic capacity on the urban road network proves that the motor vehicle has a high requirement on the road capacity, and cannot be changed in the near future. Therefore, the research of urban traffic signal control system based on intelligent integration is of high academic value. The intelligent control is designed to meet the needs of the rapid development of the city, which also has a strong practical value.

\section{Integrated design of intelligent traffic light system}

The crossroads traffic control system model is designed to make use of a set of red, green, yellow light to control the traffic intersection traffic. When the system is started and initialized, by setting the collected pedestrian and road in different locations of the pressure sensor and flow information are processed separately, and then output the analog to digital conversion, and preset control content were compared, and the formation of control scheme, the final display to give two tips for travelers. Intelligent traffic lights must be based on traffic data of the real-time traffic information, traffic and historical data to calculate the optimal control of traffic lights, traffic lights automatically adjust the signal cycle and distribution proportion, in order to realize intelligent control of traffic lights. The core module of traffic signal control system is the data analysis module, its main function is to real-time road condition information and the current traffic history information analysis, the analysis process requires the use of intelligent partition and dynamic programming algorithm. The basic model of intelligent traffic control system is shown in Figure 1, which contains the vehicle detection module, the traffic control module and the signal display module.

\begin{tabular}{|c|c|c|c|}
\hline $\begin{array}{c}\text { Vehicle detection } \\
\text { module }\end{array}$ & $\begin{array}{c}\text { Traffic control } \\
\text { module }\end{array}$ & $\begin{array}{c}\text { Signal display } \\
\text { module }\end{array}$ \\
\cline { 3 - 4 }
\end{tabular}

Fig. 1: Integrated diagram of the intelligent traffic light system

\section{Modules design of intelligent traffic light system}

\subsection{Vehicle detection module}

According to the image information transmitted by the processing, the module detects the traffic flow. Traffic flow detection module which has a fixed value judgment, the decision value is used to detect the scope of the provisions of the region have no vehicle to travel over. If there is a vehicle, the traffic flow detection module will produce a pulse signal, and the pulse signal to the traffic flow statistics module for data processing. Our traffic flow detection algorithms are image processing and simulation can be obtained, each frame of the video image pixel information, because the data can only identify the file storage platform, data pieces can be processed video image pixel information stored in 
the storage, the data can be used as the input excitation simulation testing the module of traffic flow, the pixel video image information is to be detected. When the traffic flow detection module receives the video switching pulse counter module, start to start counting, when the counter count stop switch module, the arrival of video pulse until the next. When the accumulator vehicle flow detection module in the received pulse counter counting module, accumulator of parallel gray pixel information of each video frame update by a cumulative processing, when the accumulator output end to the sub-tractor; the subtracted function will be a frame of video image gray cumulative results of current video frame with the register storage results poor treatment, the difference results will be output to the comparator; and the difference of setting threshold were compared. If the difference is greater than the set threshold, the traffic flow detection module will output a high pulse detection line.

\subsection{Traffic control module}

The main function of the traffic control module is to count the vehicles according to the traffic flow detection module. The role of the module is responsible for the statistics within the specified time range through the detection of the number of vehicles, and the output of the corresponding sampling pulse. Similarly, when the traffic control module receives an effective clear pulse, the counter in the module is cleared. In the effective detection of pulse interval, the traffic flow of the numerical value and the same time the sampling pulse output to the signal lamp control module for data processing. When the effective marker to mark invalid detection, detection signal down has not come, detected falling edge sends a high pulse, and the pulse high hand without delay register directly as the sampling pulse output, on the other hand after a delay registers a clock delay after the high pulse as a reset signal traffic counter. The signal lamp control module is designed according to the traffic control system based on the provisions of the number of sampling pulse time range of traffic sampling, a decision to east-west direction crossroads signal green time. When the traffic control traffic flow module for transmission over the sampling pulses, the signal lamp control module for sampling processing on the transmitted traffic data, the data obtained by a delay register, and then sent to the selector according to the sampled traffic data query predefined fuzzy control table, get the next cycle of the same phase green time. When the same phase green time counter starts to count and the upper limit is by querying the fuzzy control table to control the time counter, green time from the beginning to the count threshold, high light control signal output to the external hardware circuit, signal lamp, real-time distribution on the green time. When the green light is accumulated to the counter threshold, the yellow light will start to work.

\subsection{Signal display module}

The intersection traffic system in the city is mostly used to control the timing, which will inevitably lead to a road traffic volume that is large but to wait for the red light, while another empty road or traffic is relatively less but long time the 
green light, this not only allows the driver and passengers Voices of discontent., of manpower and material resources is a waste of resources. Therefore, the intelligent traffic light control system to ensure the smooth flow of road traffic is more and more serious problems for the current have good relief, especially for the car more and more traffic problems become more and more serious conditions, the system is to provide an intelligent solution for city traffic light intersection traffic control, intelligent control is the inevitable trend of development, but also the need of social development. LED digital tube has the advantages of simple structure, high luminous efficiency, low production cost, stable performance and high efficiency, long service life, bright color, easy to observe, affordable, costeffective can reduce the number of the interface. At the same time, LED, the energy saving safety and environmental advantages will use a total of negative digital dynamic tubes to display and drive. We can complete the friendly interaction between the user and the real-time image, the interface is simple and beautiful, easy to operate. Users only need to operate in accordance with the specified button, and the user can judge their own window to see whether the results are correct, you cannot grasp the relevant knowledge of the identification model. Therefore, from the point of view of operation, the development of this system is feasible.

\section{Simulation of intelligent traffic light system}

Compared with the traditional traffic control system, the biggest innovation of the intelligent traffic control system compared is to achieve signal traffic in opposite directions. It is not synchronized control. It will adjust the traffic signal control strategy according to the change of the traffic flow, to maximize the efficiency of traffic crossroads traffic. The whole control circuit consists of two control modules: the straight direction control module and the left turn direction control module. When the control program is running, the two modules are alternately operated, and the loop control is in the same direction as the left direction and the four directions of the signal in the direction of the left hand in the same direction of the four directions. The specific phase of the direction is shown in Figure 2. The specific signal distribution is shown in Table 2 and Table 3.

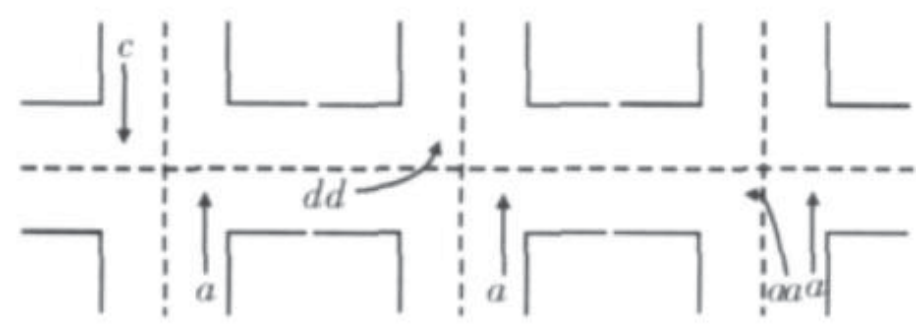

Fig. 2: Phase diagram of signals 
Table 2 Input signals of the intelligent traffic light system

\begin{tabular}{|c|c|c|c|c|}
\hline \multicolumn{5}{|c|}{ Input signals } \\
\hline Junction & $\mathrm{a}$ & $\mathrm{b}$ & $\mathrm{c}$ & $\mathrm{d}$ \\
\hline Direction & straight left & straight left & straight left & straight left \\
\hline
\end{tabular}

Table 3 Output signals of the intelligent traffic light system

\begin{tabular}{|c|l|l|l|l|}
\hline \multicolumn{5}{|c|}{ Output Signals } \\
\hline Junction & $\mathrm{a}, \mathrm{b}, \mathrm{c}, \mathrm{d}$ & $\mathrm{a}, \mathrm{b}, \mathrm{c}, \mathrm{d}$ & $\mathrm{a}, \mathrm{b}, \mathrm{c}, \mathrm{d}$ & \multicolumn{1}{c|}{$\mathrm{a}, \mathrm{b}, \mathrm{c}, \mathrm{d}$} \\
\hline Direction & Straight & Left & Right & Pedestrians \\
\hline \multirow{3}{*}{ Signals } & Green & Green & Green & Green \\
\cline { 2 - 5 } & Yellow & Yellow & Yellow & Yellow \\
\cline { 2 - 5 } & Red & Red & Red & Red \\
\hline
\end{tabular}

\section{Conclusion}

The system focuses on the problem of urban traffic congestion. In the current economic development trend of the society, the number of cars is increasing and the traffic congestion problem cannot be avoided. Therefore, the market prospect of intelligent traffic system can be expected to be very broad. At present, there are many features to be improved.We believe that after a gradual improvement, as well as the rationalization application of the technology, the system efficiency can be further improved.

\section{References}

[1]Wang Xiaojuan, Design of Intelligent Traffic Light Control System Based on PLC, Mechatronics, (3), pp. 68-72, 2015.

[2]Tan Jixiang, Ding Yu, Zhang Jie, Hua Ting, Wang Liu, The Design and Implementation of Intelligent Traffic Light ControlSystem, Computer \& Network, (8), pp. 57-59, 2015.

[3]Wang Weisong, Wang Jinsheng, Zhang Wei, Liang Qi, FPGA Implementation of the Intelligent Traffic Light Control System for Crossroads, 25(9), pp. 37 $39+44,2012$.

[4] Wu Ning, Shang Poli, Design and Implementation of Intelligent Zebra Crossing Traffic Light Control System, Mechanical Research \& Application, 26(4), pp. 179-180+187, 2013. 